\title{
Identification of Chromatin Accessibility During The Early Stage of Pig SCNT Embryo Reprogramming by ATAC-Seq
}

Xue Zhang ( $2017205006 @ n j a u . e d u . c n$ )

Nanjing Agricultural University https://orcid.org/0000-0002-9015-0517

Juan Li

Nanjing Agricultural University

Ruixin Tao

Nanjing Agricultural University

Aiwen Jiang

Nanjing Agricultural University

Changyin Zhou

Nanjing Agricultural University

\section{Hongxia Xu}

Nanjing Agricultural University

Weijian Li

Nanjing Agricultural University

Xueqing Meng

Nanjing Agricultural University

Liangliang Zhang

Nanjing Agricultural University

Xuan Zhang

Nanjing Agricultural University

Wangjun Wu

Nanjing Agricultural University

Jingli Tao

Nanjing Agricultural University

Honglin Liu

Nanjing Agricultural University

\section{Research}

Keywords: oocyte, SCNT, handmade cloning, reprogramming, ATAC-seq 
Posted Date: November 9th, 2020

DOI: https://doi.org/10.21203/rs.3.rs-102477/v1

License: (c) (i) This work is licensed under a Creative Commons Attribution 4.0 International License. Read Full License 


\section{Abstract}

Background: Somatic cell nuclear transplantation (SCNT) can transform highly differentiated donor nuclei into pluripotent nuclei through the large-scale reprogramming of chromatin. The reprogramming of chromatin has been documented to take place in the first few hours after SCNT embryo activation. Thus, studies that characterize dynamic changes in chromatin during the first few hours after embryo activation could provide insight into the mechanism and significance of genome-wide reprogramming. However, few studies have examined the epigenetic remodeling of reconstructed embryos during the early stage of reprogramming.

Results: We conducted ATAC-seq on 50 porcine SCNT-HMC embryos and 50 parthenogenetic activation (PA) embryos $10 \mathrm{~h}$ after activation. Along with pig embryonic fibroblast (PEF) ATAC-seq data, we found low levels of chromatin accessibility and gene transcription in SCNT and PA embryos. Moreover, PEF genes and the $X$ chromosome became inaccessible during embryo reprogramming. $G O$ enrichment analysis revealed that the molecular functions related to accessible chromatin in embryos primarily included transcriptional regulatory activity and SMAD binding. The differentially accessible chromatin sites between SCNT and PEF were primarily related to transcriptional activity and histone modification.

Conclusions: Despite the tight chromatin structure during the early stage of embryo reprogramming, some accessible chromatin sites, which were primarily distributed in the intergenic region, were still detected. Dynamic changes in chromatin accessibility during reprogramming were primarily related to transcriptional activity and histone modification. Generally, this study provided new insight into the dynamics and importance of chromatin accessibility during the early stages of embryo reprogramming.

\section{Background}

Mammalian DNA is wrapped around a 147-bp histone octamer and is packaged into nucleosomes. The nucleosomes further fold, condense, and form chromosomes with the aid of other structural proteins[1, 2]. DNA replication, transcription, and repair require the unpackaging of this high-level structure and the accessibility of binding sites for regulatory proteins and transcription factors[3]. The accessibility of chromatin is a highly dynamic property that plays an essential role in establishing and maintaining cellular identity [4, 5].. Because chromatin accessibility is key for the regulation of gene expression[6, 7], mapping the genome-wide accessibility of chromatin could provide insights into the regulatory mechanisms underlying global patterns of gene expression.

The most frequently used methods to detect accessible chromatin include DNase-seq and MNasesEq. However, these methods are time-consuming and labor-intensive and require tens of thousands of samples[8, 9]. In 2013, William J. Greenleaf developed a new method-ATAC-seq-an assay for transposase-accessible chromatin using sequencing. ATAC-seq is a rapid and sensitive method for integrative epigenomic analysis based on direct in vitro transposition of sequencing adaptors into native chromatin and only requires 500-50,000 cells[10]. Moreover, the experimental procedures and operation 
of ATAC-seq are simpler and more time efficient[11, 12]. In recent years, an increasing number of studies have used ATAC-seq, and this method has greatly improved since its initial development. For example, one study in 2016 used only 200 mouse embryos to perform ATAC-sEq. In 2018, the number of cells needed to perform ATAC-seq was reduced to $20[13,14]$. Such improvements make it possible to apply ATAC-seq to the study of oocytes and embryos, samples that are not easily obtained in large quantities.

Somatic cell nuclear transplantation (SCNT) can transform a highly differentiated donor nucleus into a pluripotent nucleus. After numerous divisions and differentiation steps, the reconstructed embryo finally forms a complete multicellular organism. Radical changes in gene expression patterns in cells are critically important for SCNT[15, 16]. Chromatin nucleosome positioning has been reported to undergo large-scale dynamic reorganization in the first few hours after the activation of the SCNT embryo [17], suggesting that chromatin has already been reprogrammed at the early stage of embryo activation. Therefore, studies examining the dynamic changes of chromatin in the first few hours after embryo activation could provide insight into the mechanisms and significance of genome-wide reprogramming. Although the traditional method of somatic cell nuclear transfer through a micromanipulator is still commonly used, an increasing number of researchers have used the enucleation technique of handmade cloning (HMC), which is performed using a bisection blade [18, 19]. SCNT-HCM does not require the use of expensive equipment, which not only reduces costs but also ensures a high development rate of reconstructed embryos [20,21]. However, the number of reconstructed embryos obtained by either method is highly limited, and this limitation has greatly restricted research on the epigenetic remodeling of reconstructed embryos at the genome-wide level.

Owing to their numerous physiological similarities with humans and the ease with which their cells can be obtained, pig embryos have become ideal models for studying human embryonic development [22, 23]. Here, global dynamic changes in chromatin during the early stage of embryo reprogramming were explored by ATAC-seq using 50 porcine SCNT-HMC embryos and 50 parthenogenetic embryos that were collected $10 \mathrm{~h}$ after activation. Along with pig embryonic fibroblast (PEF) ATAC-seq data, we mapped the accessible chromatin distribution during the early stage of embryo reprogramming. The goal of this study was to characterize dynamic changes in chromatin accessibility and the importance of genome-wide chromatin accessibility in the first few hours of embryo programming.

\section{Methods}

\section{Oocyte collection and in vitro maturation (IVM)}

Pig ovaries were obtained from a local slaughterhouse and were transported to the laboratory in $0.9 \%$ $\mathrm{NaCl}$ containing $800 \mathrm{IU} / \mathrm{ml}$ of gentamicin at $37^{\circ} \mathrm{C}$. Cumulus-oocyte complexes (COCs) were collected by vacuum suction of 3-8 $\mathrm{ml}$ of follicle fluid. After washing, the COCs with compact cumulus cells and a uniform ooplasm were cultured in four-well dishes with IVM medium at $38.5^{\circ} \mathrm{C}$ in a $5 \% \mathrm{CO}_{2}$ atmosphere with saturated humidity. 


\section{Parthenogenetic activation (PA)}

After 42-44 h of IVM, COCs were digested using hyaluronidase ( $1 \mathrm{mg} / \mathrm{ml}$ in TCM-199 culture medium) to obtain denuded oocytes (DOs). Oocytes with the first polar body were selected and placed into a microslide $0.5-\mathrm{mm}$ fusion chamber with activation solution ( $\mathrm{F}^{+}$consisted of $0.3 \mathrm{M}$ mannitol, $0.5 \mathrm{M}$ HEPES, $0.1 \mathrm{M} \mathrm{MgSO}_{4} \cdot 7 \mathrm{H}_{2} \mathrm{O}$, and $0.1 \mathrm{M} \mathrm{CaCl}_{2}$ ). Oocytes were electrically activated using a single direct current (DC) pulse of $0.9 \mathrm{kV} / \mathrm{cm}$ for $120 \mathrm{~ms}$ (CRY-3B Cell fusion instrument, Ningbo, China). Next, chemical activation was performed with cytochalasin $B(C B, 5 \mathrm{mg} / \mathrm{ml})$ and cycloheximide (CHX, 1 $\mathrm{mg} / \mathrm{ml}$ ) in PZM-3 medium for $4 \mathrm{~h}$. After chemical activation, embryos were transferred to PZM-3 medium and cultured at $38.5^{\circ} \mathrm{C}$ in an atmosphere of $5 \% \mathrm{CO}_{2}$ with saturated humidity.

\section{SCNT-HMC: oriented handmade enucleation, two-step fusion, and activation}

The basic manipulations of HMC have been previously described[24]. Briefly, after 42-44 h of IVM, DOs were treated with pronase $(3.3 \mathrm{mg} / \mathrm{mL}$ in TCM-199 with $33 \%[\mathrm{v} / \mathrm{v}]$ cattle serum) to remove the zona pellucida. DOs were then washed with T2 (TCM-199 with 2\% [v/v] cattle serum) and T20 (TCM-199 with $20 \%[\mathrm{v} / \mathrm{v}]$ cattle serum), and were transferred to a T2 drop with $2.5 \mathrm{mg} / \mathrm{mL}$ cytochalasin B. Oocytes were rotated by a fire-polished glass capillary to observe the polar body. Oocytes were then bisected with a microblade. After the bisection, the halves of the cytoplasts of oocytes without polar bodies were selected and transferred to $\mathrm{T} 2$ drops for further fusion.

Approximately 200 PEF donor cells were prepared in a T2 drop after trypsinization. All of the cytoplasts of the oocytes were incubated in T10 (TCM-199 with 10\% [v/v] cattle serum) drops for a short period, transferred individually to $1 \mathrm{mg} / \mathrm{mL}$ phytohemagglutinin for 2-3 s, and then were dropped over single donor cells in T2 drops to form cytoplast-PEF pairs. The pairs were then aligned to one wire of a fusion chamber (BTX) by an alternating current of $0.06 \mathrm{kV} / \mathrm{cm}$ and $700 \mathrm{kHz}$, followed by fusion with a single DC impulse of $2.0 \mathrm{kV} / \mathrm{cm}$ for $9 \mathrm{~ms}$ by an electrofusion machine (BLS). After $1 \mathrm{hr}$ of incubation in T10 drops, the successfully fused pairs were selected and transferred into the activation medium. The experimental manipulations for the second fusion were similar to those of the first fusion described above, except that the DC impulse was $0.86 \mathrm{kV} / \mathrm{cm}$ for $80 \mathrm{~ms}$. Fusion was again observed in a T10 drop after a 15-min incubation period. We used chemical activation after the two-step fusion. The selected, successfully fused, and reconstructed embryos were then transferred to porcine zygote medium-3 culture solution for further culture.

\section{ATAC-seq DNA library preparation and sequencing}

A total of 50 GV DOs, 50 PA embryos cultured $10 \mathrm{hr}$ after activation, and 50 SCNT embryos cultured $10 \mathrm{hr}$ after activation were collected for ATAC-seq. The miniATAC-seq libraries were prepared following previously published methods $[13,14]$ with modifications to mitochondrial clearance. A filter membrane with a 1.2- $\mu \mathrm{m}$ pore size (\#RNWP02500, Merck) was used to separate mitochondria and nuclei. The filter membrane was placed in a centrifuge tube. Pre-cooled lysis buffer (\#NUC101, Sigma) was added, and care was taken to ensure that the tube was full of lysis buffer above and below the filter membrane. 
Oocytes and embryos were treated with pronase $(3.3 \mathrm{mg} / \mathrm{mL}$ in TCM-199 with $33 \%[\mathrm{v} / \mathrm{v}]$ cattle serum) to remove the zona pellucida, which were then transferred into lysis buffer above the filter membrane. Lysed oocytes or embryos were placed on ice for $5 \mathrm{~min}$, followed by centrifugation at $12000 \mathrm{~g}$ for $5 \mathrm{~min}$ at $4^{\circ} \mathrm{C}$. The mixture above the filter membrane was then carefully collected and transferred into a new EP tube. After centrifugation at $500 \mathrm{~g}$ for $5 \mathrm{~min}$ at $4{ }^{\circ} \mathrm{C}$, the supernatant containing the mitochondria was discarded, and the precipitate containing the nucleus was used for DNA library construction.

After mitochondrial clearance, $6 \mu$ of lysis buffer, $4 \mu \mathrm{l}$ of ddH2O, $4 \mu \mathrm{l}$ of $5 \times \mathrm{TTBL}$, and $5 \mu \mathrm{l}$ of TTE mix V 5 (TD502, Vazyme) were added to the sample, followed by mixing and incubation at $37^{\circ} \mathrm{C}$ for $30 \mathrm{~min}$. The stop buffer was then added to end the tagmentation. Next, PCR was performed to amplify the library for 18 cycles for approximately 50 oocytes or embryos $\left(72^{\circ} \mathrm{C}\right.$ for 3 min; $98^{\circ} \mathrm{C}$ for $30 \mathrm{~s}$; thermocycling at 98 ${ }^{\circ} \mathrm{C}$ for $15 \mathrm{~s}, 60^{\circ} \mathrm{C}$ for $30 \mathrm{~s}$, and $72{ }^{\circ} \mathrm{C}$ for $3 \mathrm{~min}$; and $72{ }^{\circ} \mathrm{C}$ for $5 \mathrm{~min}$ ). Finally, libraries were purified with DNA Clean Beads (Vazyme) and were subjected to next-generation sequencing by Annoroad Gene Tech. A schematic representation of ATAC-seq for oocytes and embryos was provided in Figure 1A, and two replicates were performed for each group.

\section{ATAC-seq data analysis}

Raw data (raw reads) of fastq format were processed, and Trimmomaitic (v0.36) was used to remove adapter sequences and poor-quality reads to obtain the clean data (clean reads). Bowtie 2 was used to map the clean data to the reference genome. Peaks corresponding to the open region in the genome were detected by MACS2. Gene ontology (GO) enrichment analysis was performed using the GOseq R package, and gene length bias was corrected. GO terms with corrected $P$ values less than 0.05 were considered significantly enriched for peak-related genes.

\section{Results}

\section{Removal of mitochondria from oocytes and embryos}

To demonstrate that mitochondrial clearance was effective, we first attempted mitochondrial removal with 100 mouse oocytes. According to the methods (Figure 1A) described above, 100 oocytes without zona pellucida were transferred into lysis buffer above the filter membrane. After lysis and centrifugation, the mixture above the filter membrane and the mixture below the filter membrane were collected for DNA extraction. PCR was then performed to detect the efficiency of mitochondrial clearance. Cxcr3 (F: CGTGCACTATGCTCAGATATCTGTC; R: CCACAGGATTTCAGCCTGAACTTTG) was chosen to represent the nucleus (Nu), and the mitochondrial marker gene ND2 (F: CAACCATTTGCAGACGCCAT; R: TTGGGCTACGGCTCGTAAAG) was used to represent the mitochondria (Mt). The agarose gel electrophoresis results (Figure 1B) showed that both the nucleus and mitochondria were present in the upper mixture, whereas only the mitochondria were present in the mixture below. This result demonstrates that part of the mitochondria could be filtered out, but the nucleus could not pass through the filter membrane. To further test the efficiency of this method to remove mitochondria, we sequenced 50 pig oocytes. Before mitochondrial clearance, the proportion of non-nuclear DNA was as high as $78.61 \%$, 
whereas the proportion of nuclear DNA was only $21.93 \%$ (Figure 1C). After mitochondrial clearance, the proportion of non-nuclear DNA was reduced to $27.51 \%$, whereas the proportion of nuclear DNA was increased to $72.49 \%$. Figure 1D shows the ratio of nuclear DNA and non-nuclear DNA in the final samples (GV1: Nucleus: 49.72\%, Non-nucleus: 50.28\%; GV2: Nucleus: 72.83\%, Non-nucleus: 27.17\%; PA1: Nucleus: 73.90\%, Non-nucleus: 26.10\%; PA2: Nucleus: 61.21\%, Non-nucleus: 38.79\%; SCNT1: Nucleus: 44.51\%, Non-nucleus: $55.49 \%$; and SCNT2: Nucleus: $62.37 \%$, Non-nucleus: $37.63 \%)$. Thus, mitochondria were effectively removed from oocytes and embryos.

\section{Accessible chromatin landscape during an early stage of embryo reprogramming}

A total of 50 GV oocytes, 50 PA embryos, and 50 SCNT embryos were collected for ATAC-seq analysis, and two replicates were performed for each group. Figure 2A shows the randomly selected IGV browser view, which confirmed that the repeatability between replicates was satisfactory. To study the dynamic changes in the accessibility of chromatin sites at an early stage of embryo reprogramming, our data were also analyzed along with the ATAC-seq data from PEF (unpubl. data). First, we counted the average number of peaks of replicated samples in each group; the results are shown in Figure 2B. A total of 11152 peaks were enriched in PA, 3710 peaks in SCNT, 64634 peaks in GV, and 68019 peaks in PEF. These results suggested that fewer accessible regions were present in PA and SCNT embryos compared with GV oocytes and PEF. Next, we calculated the coverage ratio of peaks in the genome (Figure 2C) and found that PEF had the highest coverage ratio, whereas PA and SCNT had much lower ratios than PEF and GV. These results indicated that low levels of chromatin accessibility and expression of the genome were present in embryos $10 \mathrm{~h}$ after parthenogenetic activation and $10 \mathrm{~h}$ after somatic cell nuclear transfer.

\section{Dynamic changes in chromatin accessibility around transcription start sites (TSSs)}

To explore changes in chromatin accessibility during the somatic cell reprogramming of genes with different expression levels, we downloaded PEF RNA-seq data (accession number: GSM595679) from the NCBI (National Center for Biotechnology Information) database. A total of 24322 genes were divided into three categories based on their level of expression as indicated by the RNA-seq data: top $5 \%$ highest genes (1217 genes), silent genes (8229 genes), and other genes (14875 genes). We then analyzed the chromatin accessibility near the TSS of these three types of genes and used deptools for visual drawings. The results are shown in Figure 3. For the top 5\% highest genes, there was a strong enrichment signal near the TSS in PEF cells, but there was no obvious enrichment signal near the TSS in SCNT, PA, and GV (Figure 3A). For silent genes, the enrichment signal peaks near the TSS were significantly reduced in PEF cells, but there were no obvious enrichment signal peaks near the TSS in SCNT, PA, and GV (Figure 3B). Other types of genes also had enrichment peaks near the TSS in PEF cells, but the enrichment level was not as high as that observed in the $5 \%$ highest genes. Other types of genes had enrichment peaks near the TSS in SCNT, PA, and GV, but they were not typical enrichment peaks of ATAC-seq (Figure 3C). Thus, the chromatin of the highly expressed PEF gene had strong accessibility near the TSS region in PEF cells, but these accessible sites were inaccessible during embryo reprogramming.

\section{Dynamic changes in $\mathrm{X}$ chromatin accessibility during embryo reprogramming}


Given that the $X$ chromosome experienced a large change during the early stage of reprogramming $[25$, 26], we analyzed the dynamic changes in $X$ chromatin accessibility near the TSS during somatic cell reprogramming. Figure 4 shows the $X$ chromosome accessibility near the TTS in PEF, SNCT, PA, and GV samples. We found that there was a strong enrichment signal near the TSS region in PEF cells, but no obvious enrichment signal was present in GV, PA, and SCNT. This finding suggested that X chromatin had high accessibility in PEF cells but was tightly structured in oocytes and embryos and had no transcriptional activity.

\section{Distribution of chromatin accessibility sites in gene functional areas}

Although the chromatin in the embryo was relatively tight, some accessibility sites could still be detected. To further explore the roles of these accessibility sites, we next calculated the distribution of chromatin accessibility sites in gene functional areas. In PEF, the distribution of accessible chromatin in the promoter area accounted for $8.99 \%$ of the functional gene area, $5^{\prime}$ UTR accounted for $3.05 \%$, and the exon area accounted for $7.22 \%$ (Figure $5 \mathrm{~A}$ ). However, the proportion of accessible chromatin in the promoter region was decreased in SCNT, PA, and GV (Figure 5B, C, D), which was $3.14 \%, 3.42 \%$, and $3.24 \%$, respectively. The proportion of accessible chromatin in the $5^{\prime}$ UTR in the promoter region was also decreased, which was $0.43 \%, 0.54 \%$, and $0.59 \%$ in SCNT, PA, and GV, respectively. The proportion of accessible chromatin in the exon region was also decreased to $4.46 \%, 4.49 \%$, and $4.63 \%$ in SCNT, PA, and $\mathrm{GV}$, respectively. Moreover, we also found that the proportion of chromatin open sites in the intergenic region was increased to $58.15 \%, 52.98 \%$, and $46.63 \%$ in SCNT, PA, and GV respectively (Figure 5B, C, D), whereas the proportion was only $38.57 \%$ in PEF (Figure $5 \mathrm{~A}$ ). Thus, the ratio of chromatin accessibility sites decreased in gene regions but increased in intergenic regions during an early stage of reprogramming.

\section{The molecular function of accessible chromatin sites}

We suspect that the molecular function of the cell changes as highly differentiated oocytes change to pluripotent embryos. To further characterize the role of accessible chromatin sites in oocytes and embryos, we performed gene ontology (GO) enrichment analysis on GV oocytes, PA embryos, and SCNT embryos and focused on the molecular function (MF). The top 10 results are shown in Figure 6. According to the $\mathrm{GO}$ annotation, MF terms in GV oocytes included chromatin binding, actin binding, cadherin binding, cholesterol binding, transforming growth factor beta receptor binding, and 3',5'-cyclicnucleotide phosphodiesterase (PDE) activity. MF terms in PA oocytes primarily included PDE activity, transcription coregulator activity, translation regulator activity, collagen binding, transcription factor binding, and SMAD binding. MF terms for SCNT primarily included PDE activity, translation regulator activity, translation regulator activity, collagen binding, SMAD binding, and protein-containing complex binding. Translation regulator activity and SMAD binding were recovered in both PA and SNCT embryos, and PDE activity was recovered in both oocytes and embryos. 
To investigate the biological function of chromatin accessibility dynamics during somatic cell reprogramming, the different peaks between SCNT and PEF were analyzed for GO enrichment. Here, we compared PEF1 with SCNT because PEF1 was from the same cell line and the same generation of SCNT nuclear donor cells, thereby minimizing the possible effects caused by differences in cell origin and generation. Figure 7 shows the results of the $\mathrm{GO}$ enrichment analysis including the biological process (BP) category, cellular component (CC) category, and molecular function (MF) category. The top 10 terms are shown for each category (Figure 7). BP terms indicated that the different accessible chromatin sites between SCNT and PEF were involved in histone modification, blastocyst formation, and covalent chromatin modification. CC terms indicated involvement with the transferase complex, transcriptional repressor complex, and transcription factor complex. MF terms indicated involvement with DNA-binding transcription factor activity, transcription coregulator activity, transcription factor binding, and histone binding. Thus, changes in chromatin accessibility were primarily related to transcriptional activity and histone modification at an early stage of embryo reprogramming.

\section{Discussion}

Mammalian oocytes and preimplantation embryos contain high copy numbers (at least 100,000 copies) of mtDNA that are completely accessible[27, 28]. Therefore, the large amount of mtDNA in ATAC-seq data poses a major obstacle for profiling accessible chromatin in oocytes and embryos. To address this obstacle, we performed mitochondrial clearance before establishing the DNA library. According to previous studies on cell nuclear and cytoplasmic separation, treatment with cytochalasin B (CB) and high-speed centrifugation can separate the nucleus and cytoplasm, and the separated cytoplasm can remain active[29]. In this study, we used lysis buffer in the place of $C B$, used a 1.2- $\mu$ m pore size filter, and centrifuged at high speed to separate cytoplasm and mitochondria. Our results indicated that the amount of mtDNA in oocytes and embryos had been successfully reduced.

The number of signal peaks can reflect chromatin accessibility. Few signal peaks were observed in the genomes in PA and SCNT embryos, suggesting a low level of chromatin accessibility and gene transcription in embryos $10 \mathrm{~h}$ after activation. Despite this low level of accessibility, some accessible chromatin sites could still be detected. However, our results suggest that these accessibility sites were not highly expressed genes in somatic cells; furthermore, the X chromosome was in an inactive state, which is consistent with previous studies [17, 30], indicating that large-scale chromatin remodeling had occurred at an early stage of embryo reprogramming. Given that inhibiting the small-scale transcription of the zygotic genome before activation can lead to the arrest of embryonic development [31], we speculate that accessible chromatin in the embryo $10 \mathrm{~h}$ after activation may be related to subsequent embryonic developmental events.

To verify this hypothesis, we determined the distribution of accessible chromatin in the functional regions of the genome in the embryo. We found that the accessible chromatin in the embryo $10 \mathrm{~h}$ after activation was primarily distributed in the intergenic region, and the proportion of accessible chromatin in the promoter region, 5' UTR, and exons was low. This finding also indicated that there was a low level of 
transcriptional activity in embryos during the early stages of reprogramming. Some studies have suggested that the intergenic region plays a role in remodeling chromatin structure[32] and that largescale chromatin remodeling takes place during the process of embryo reprogramming[33]. We suggest that the accessible chromatin in the embryo $10 \mathrm{~h}$ after activation may be related to the regulation of chromatin structure. Consistent with this suggestion, the results of the $\mathrm{GO}$ enrichment analysis indicated that both PA and SCNT were enriched in translation regulator activity and SMAD binding. SMAD transcription factors are central to the transforming growth factor-beta (TGFbeta) pathway, and their activation and deactivation are closely related to the assembly of the transcription complex [34]. Because the first and most important step during the complex oocyte-to-embryo transition is zygotic genome activation [35], we speculate that these accessible chromatin sites during the early stage of embryo reprogramming may prepare oocytes for the ensuing zygotic genome activation.

To further clarify the significance of the dynamic changes in chromatin accessibility during embryo reprogramming, we performed GO enrichment analysis on the different peaks between PEF and SCNT. The dynamic changes in chromatin accessibility during somatic cell reprogramming were found to be involved in the regulation of histone modification and transcriptional activity. Given that histone modification involves epigenetic modification of the genome and that changes in epigenetic modifications alter gene expression patterns [36,37], the remodeling of chromatin accessibility during somatic cell reprogramming was driven by the regulation of gene expression patterns through epigenetic modification. However, epigenetic analysis, along with transcriptomic data, is needed to verify this possibility. Because of the limited number of samples, there are currently no comprehensive global genetic modification data on pig somatic cell nuclear transfer embryos; there is thus a need for additional, in-depth studies to be conducted.

We also found that the accessible chromatin in oocytes and embryos both involved PDE activity, which degraded CAMP, and high levels of cAMP are related to the inhibition of meiosis[38, 39]. The PA and SCNT embryos had recently completed meiosis, and this chromatin open site may be inherited from the M2 oocyte. Because mature GV oocytes were collected, no transcriptional activity was detected in GV oocytes [40]; the distribution of accessible chromatin in gene functional areas also confirmed the lack of transcriptional activity. Thus, the detected PDE activity may prepare oocytes for meiotic activation. However, determining whether this accessible chromatin was transcriptionally active will require a comprehensive analysis of epigenetic modifications and transcriptomes. Because of limitations in experimental technology and the number of samples, much work remains to be done in follow-up studies.

\section{Conclusions}

Despite the tight chromatin structure observed during the early stage of embryo reprogramming, some accessible chromatin sites, which were primarily distributed in the intergenic region, could still be detected. Dynamic changes in chromatin accessibility during reprogramming were primarily related to transcriptional activity and histone modification. Generally, this study provided new insight into the dynamics and importance of chromatin accessibility during the early stages of embryo reprogramming. 


\section{Declarations}

\section{Author contributions}

All authors were involved in designing and planning experiments, preparing and reviewing the article. Xue Zhang and Ruixin Tao performed parthenogenetic activation; Juan Li, Xue Zhang, Ruixin Tao, Aiwen Jiang, Weijian Li performed the SCNT-HMC experiments; Xue Zhang and Changyin Zhou performed the ATAC-seq DNA library preparation. Xue Zhang and Honglin Liu analysed the data; Xue Zhang and Honglin Liu wrote the article.

\section{Acknowledgments}

We thank Dr. Kai Xu from School of Life Sciences, Tsinghua University Beijing, China for the helpful suggestions on writing this manuscript.

\section{Funding}

This work was supported by the State Key Program of National Natural Science Foundation of China (No. 31630072) and the Natural Science Foundation of Jiangsu Province (BK20171372).

\section{Availability of data and materials}

All data generated or analyzed during this study are available from the corresponding author on reasonable request.

\section{Ethics approval}

All animal experiments in this study were approved by the Animal Ethics Committee at Nanjing Agricultural University (Nanjing, China).

\section{Consent for publication}

Not applicable.

\section{Conflict of interest}

The authors declare that there is no conflict of interests.

\section{References}

1. Eustermann S, Schall K, Kostrewa D, Lakomek K, Strauss M, Moldt M, Hopfner KP. Structural basis for ATP-dependent chromatin remodelling by the INO80 complex. Nature 2018; 556(7701):386-390.

2. McGinty RK, Tan S. Nucleosome structure and function. Chemical reviews 2015; 115(6):2255-2273. 
3. Fierz B, Poirier MG. Biophysics of Chromatin Dynamics. Annual review of biophysics 2019; 48:321345.

4. Klemm SL, Shipony Z, Greenleaf WJ. Chromatin accessibility and the regulatory epigenome. Nature reviews Genetics 2019; 20(4):207-220.

5. Chen Y, Tokuda JM, Topping T, Sutton JL, Meisburger SP, Pabit SA, Gloss LM, Pollack L. Revealing transient structures of nucleosomes as DNA unwinds. Nucleic acids research 2014; 42(13):87678776.

6. Agbleke AA, Amitai A, Buenrostro JD, Chakrabarti A, Chu L, Hansen AS, Koenig KM, Labade AS, Liu S, Nozaki T et al. Advances in Chromatin and Chromosome Research: Perspectives from Multiple Fields. Mol Cel/ 2020; 79(6):881-901.

7. Li B, Carey M, Workman JL. The role of chromatin during transcription. Cell 2007; 128(4):707-719.

8. Liu Y, Fu L, Kaufmann K, Chen D, Chen M. A practical guide for DNase-seq data analysis: from data management to common applications. Briefings in bioinformatics 2019; 20(5):1865-1877.

9. Teif VB. Nucleosome positioning: resources and tools online. Briefings in bioinformatics 2016; 17(5):745-757.

10. Buenrostro JD, Giresi PG, Zaba LC, Chang HY, Greenleaf WJ. Transposition of native chromatin for fast and sensitive epigenomic profiling of open chromatin, DNA-binding proteins and nucleosome position. Nature methods 2013; 10(12):1213-1218.

11. Yan F, Powell DR, Curtis DJ, Wong NC. From reads to insight: a hitchhiker's guide to ATAC-seq data analysis. Genome biology 2020; 21(1):22.

12. Baek S, Lee I. Single-cell ATAC sequencing analysis: From data preprocessing to hypothesis generation. Computational and structural biotechnology journal 2020; 18:1429-1439.

13. Wu J, Huang B, Chen H, Yin Q, Liu Y, Xiang Y, Zhang B, Liu B, Wang Q, Xia W et al. The landscape of accessible chromatin in mammalian preimplantation embryos. Nature 2016; 534(7609):652-657.

14. Wu J, Xu J, Liu B, Yao G, Wang P, Lin Z, Huang B, Wang X, Li T, Shi S et al. Chromatin analysis in human early development reveals epigenetic transition during ZGA. Nature 2018; 557(7704):256-260.

15. Matoba S, Zhang Y. Somatic Cell Nuclear Transfer Reprogramming: Mechanisms and Applications. Cell stem cell 2018; 23(4):471-485.

16. Wilmut I, Bai Y, Taylor J. Somatic cell nuclear transfer: origins, the present position and future opportunities. Philosophical transactions of the Royal Society of London Series B, Biological sciences 2015; 370(1680):20140366.

17. Tao C, Li J, Zhang X, Chen B, Chi D, Zeng Y, Niu Y, Wang C, Cheng W, Wu W et al. Dynamic Reorganization of Nucleosome Positioning in Somatic Cells after Transfer into Porcine Enucleated Oocytes. Stem cell reports 2017; 9(2):642-653.

18. Du Y, Kragh PM, Zhang Y, Li J, Schmidt M, Bogh IB, Zhang X, Purup S, Jorgensen AL, Pedersen AM et al. Piglets born from handmade cloning, an innovative cloning method without micromanipulation. Theriogenology 2007; 68(8):1104-1110. 
19. Kurome M, Ishikawa T, Tomii R, Ueno S, Shimada A, Yazawa H, Nagashima H. Production of transgenic and non-transgenic clones in miniature pigs by somatic cell nuclear transfer. The Journal of reproduction and development 2008; 54(3):156-163.

20. Kragh PM, Nielsen AL, Li J, Du Y, Lin L, Schmidt M, Bogh IB, Holm IE, Jakobsen JE, Johansen MG et al. Hemizygous minipigs produced by random gene insertion and handmade cloning express the Alzheimer's disease-causing dominant mutation APPsw. Transgenic research 2009; 18(4):545-558.

21. Li J, Li R, Villemoes K, Liu Y, Purup S, Callesen H. Developmental potential and kinetics of pig embryos with different cytoplasmic volume. Zygote 2015; 23(2):277-287.

22. Perleberg $C$, Kind A, Schnieke A. Genetically engineered pigs as models for human disease. Disease models \& mechanisms 2018; 11(1).

23. Kobayashi T, Zhang H, Tang WWC, Irie N, Withey S, Klisch D, Sybirna A, Dietmann S, Contreras DA, Webb $\mathrm{R}$ et al. Principles of early human development and germ cell program from conserved model systems. Nature 2017; 546(7658):416-420.

24. Li J, Villemoes K, Zhang Y, Du Y, Kragh PM, Purup S, Xue Q, Pedersen AM, Jorgensen AL, Jakobsen JE et al. Efficiency of two enucleation methods connected to handmade cloning to produce transgenic porcine embryos. Reprod Domest Anim 2009; 44(1):122-127.

25. Pasque V, Plath K. X chromosome reactivation in reprogramming and in development. Current opinion in cell biology 2015; 37:75-83.

26. Janiszewski A, Talon I, Chappell J, Collombet S, Song J, De Geest N, To SK, Bervoets G, Marin-Bejar O, Provenzano $\mathrm{C}$ et al. Dynamic reversal of random X-Chromosome inactivation during iPSC reprogramming. Genome research 2019; 29(10):1659-1672.

27. Shoubridge EA, Wai T. Mitochondrial DNA and the mammalian oocyte. Current topics in developmental biology 2007; 77:87-111.

28. Shoubridge EA. Mitochondrial DNA segregation in the developing embryo. Human reproduction 2000; 15 Suppl 2:229-234.

29. Veomett G, Prescott DM, Shay J, Porter KR. Reconstruction of mammalian cells from nuclear and cytoplasmic components separated by treatment with cytochalasin B. Proceedings of the National Academy of Sciences of the United States of America 1974; 71(5):1999-2002.

30. Ohhata T, Wutz A. Reactivation of the inactive X chromosome in development and reprogramming. Cellular and molecular life sciences : CMLS 2013; 70(14):2443-2461.

31. Abe KI, Funaya S, Tsukioka D, Kawamura M, Suzuki Y, Suzuki MG, Schultz RM, Aoki F. Minor zygotic gene activation is essential for mouse preimplantation development. Proceedings of the National Academy of Sciences of the United States of America 2018; 115(29):E6780-E6788.

32. Ransohoff JD, Wei Y, Khavari PA. The functions and unique features of long intergenic non-coding RNA. Nature reviews Molecular cell biology 2018; 19(3):143-157.

33. Xu Q, Xie W. Epigenome in Early Mammalian Development: Inheritance, Reprogramming and Establishment. Trends in cell biology 2018; 28(3):237-253. 
34. Massague J, Seoane J, Wotton D. Smad transcription factors. Genes \& development 2005; 19(23):2783-2810.

35. Svoboda P. Mammalian zygotic genome activation. Seminars in cell \& developmental biology 2018; 84:118-126.

36. Pal S, Tyler JK. Epigenetics and aging. Science advances 2016; 2(7):e1600584.

37. Tammen SA, Friso S, Choi SW. Epigenetics: the link between nature and nurture. Molecular aspects of medicine 2013; 34(4):753-764.

38. Pan B, Li J. The art of oocyte meiotic arrest regulation. Reproductive biology and endocrinology : RB\&E 2019; 17(1):8.

39. Gupta A, Pandey AN, Sharma A, Tiwari M, Yadav PK, Yadav AK, Pandey AK, Shrivastav TG, Chaube SK. Cyclic nucleotide phosphodiesterase inhibitors: possible therapeutic drugs for female fertility regulation. European journal of pharmacology 2020; 883:173293.

40. Christou-Kent M, Dhellemmes M, Lambert E, Ray PF, Arnoult C. Diversity of RNA-Binding Proteins Modulating Post-Transcriptional Regulation of Protein Expression in the Maturing Mammalian Oocyte. Cells 2020; 9(3).

\section{Figures}

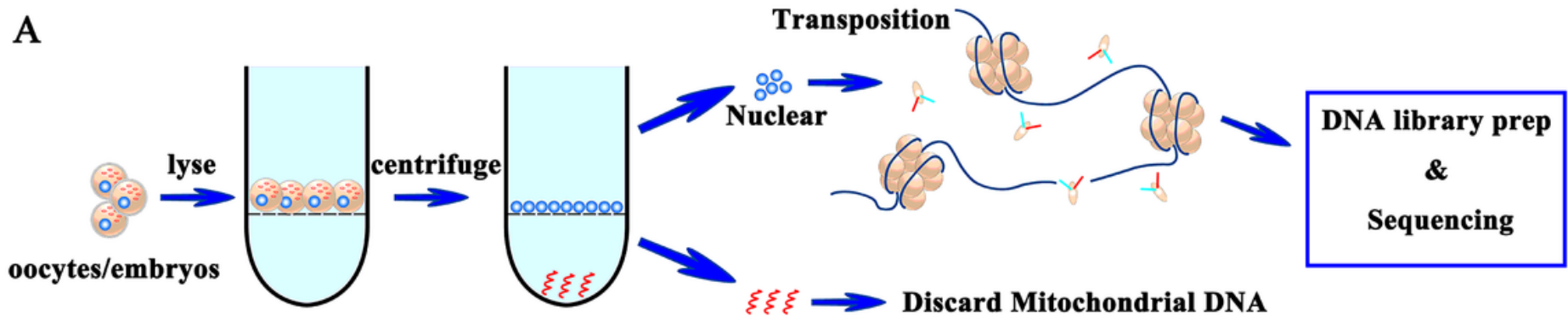

B

C

Percentage of Nuclear and Non-nuclear DNA after mitochondrial clearance

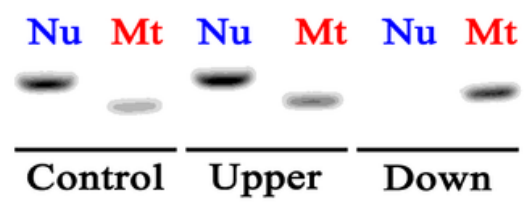

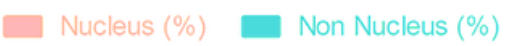

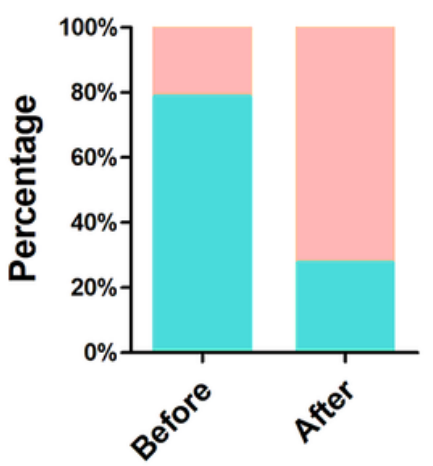

D

Percentage of Nuclear and Non-nuclear DNA in samples

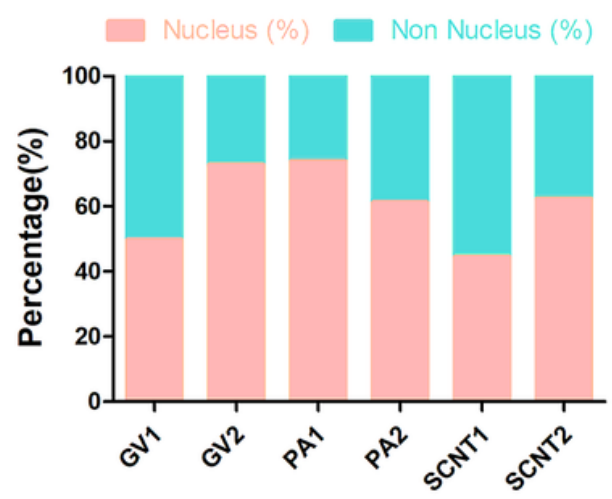


Figure 1

Mitochondrial clearance in oocytes and embryos. A. Schematic representation of ATAC-seq for oocytes and embryos. B. Agarose gel electrophoresis shows the PCR assay of the mitochondrial clearance effect. Nu: nucleus; Mt: Mitochondria; Control: without mitochondria removal; Upper: mixture above the filter membrane after centrifugation; Down: mixture below the filter membrane after centrifugation. C. Percentage of nuclear and non-nuclear DNA before and after mitochondrial clearance. D. Percentage of nuclear and non-nuclear DNA in samples after mitochondrial clearance.

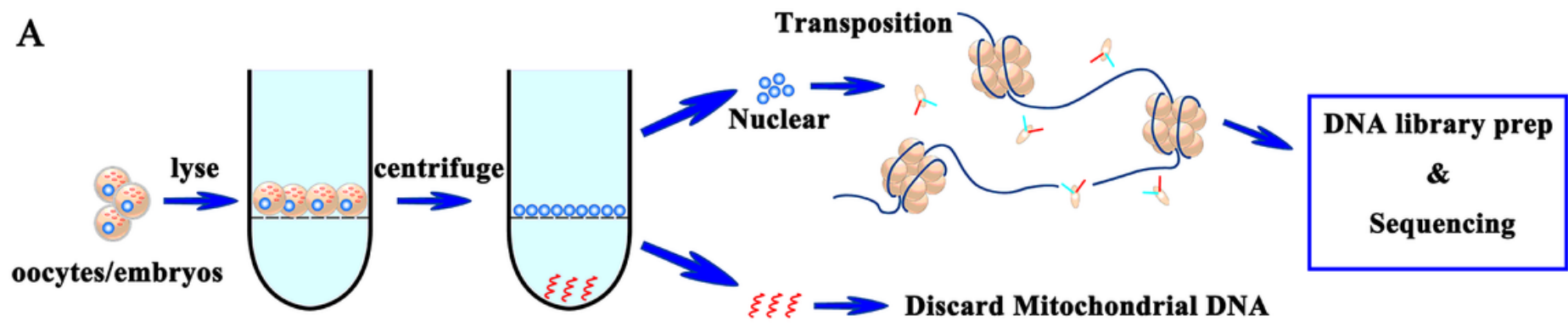

B

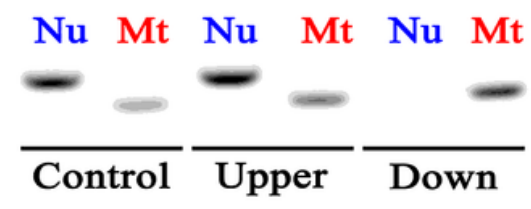

C

Percentage of Nuclear and Non-nuclear DNA after mitochondrial clearance

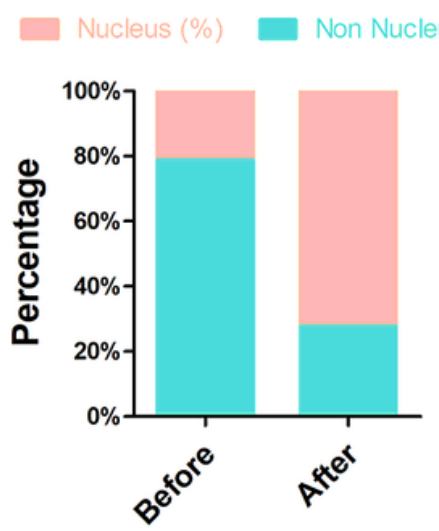

D Percentage of Nuclear and Non-nuclear DNA
in samples

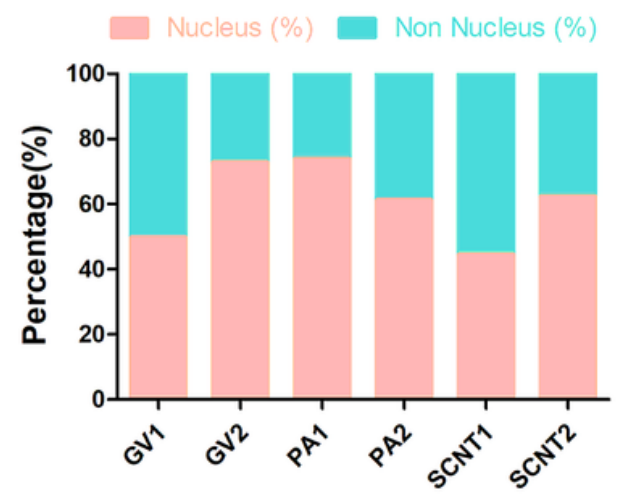

\section{Figure 1}

Mitochondrial clearance in oocytes and embryos. A. Schematic representation of ATAC-seq for oocytes and embryos. B. Agarose gel electrophoresis shows the PCR assay of the mitochondrial clearance effect. Nu: nucleus; Mt: Mitochondria; Control: without mitochondria removal; Upper: mixture above the filter membrane after centrifugation; Down: mixture below the filter membrane after centrifugation. C. Percentage of nuclear and non-nuclear DNA before and after mitochondrial clearance. D. Percentage of nuclear and non-nuclear DNA in samples after mitochondrial clearance. 
A

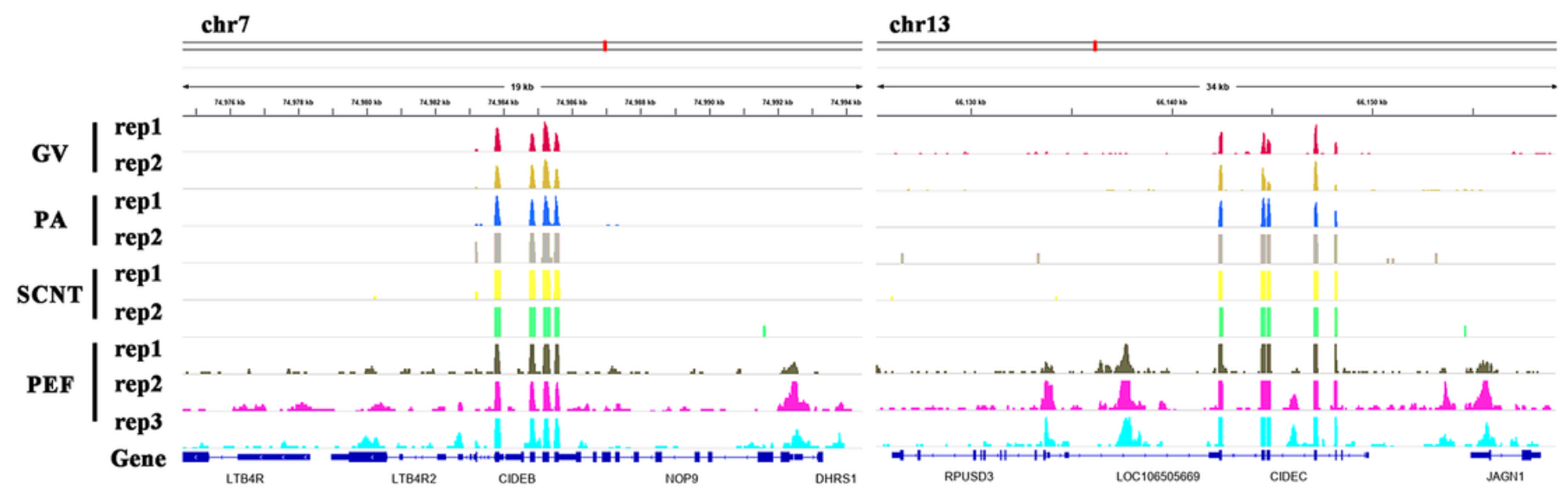

B

Peaks number

C

Coverage ratio of peaks
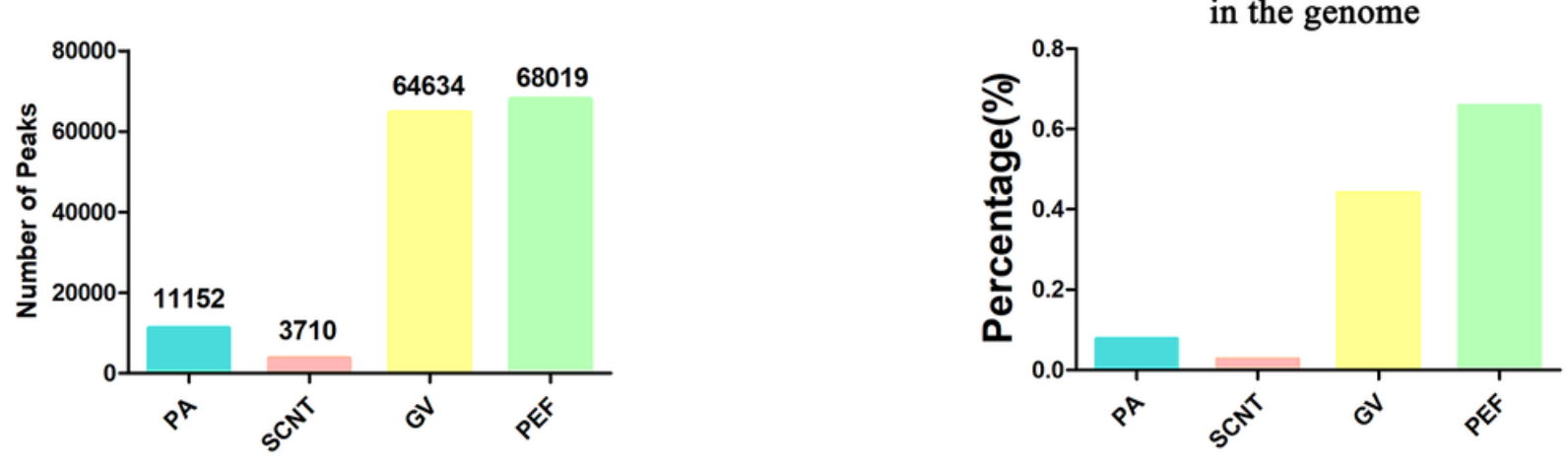

Figure 2

The accessible chromatin landscapes during embryo reprogramming. A. The IGV browser view shows the enrichment of ATAC-seq in GV, PA, SCNT, and PEF samples. Chromosomes and IGV windows were randomly selected. B. Number of accessible regions detected in four groups. C. Coverage ratio of peaks in the genome detected by MACS2. 
A

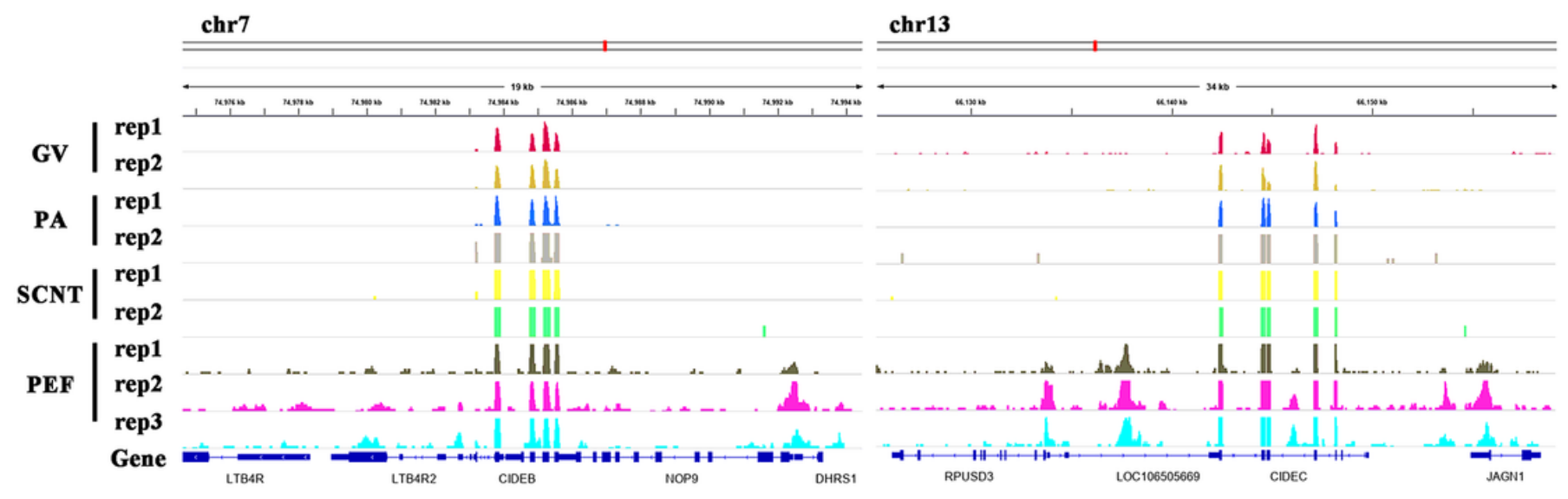

B

Peaks number

C

Coverage ratio of peaks
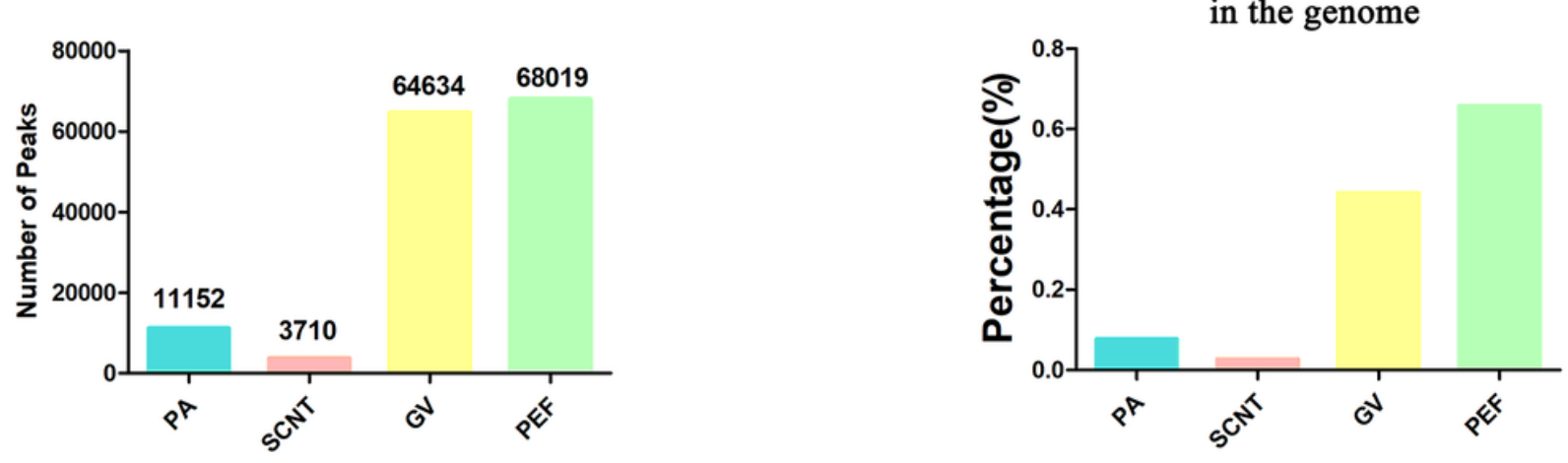

Figure 2

The accessible chromatin landscapes during embryo reprogramming. A. The IGV browser view shows the enrichment of ATAC-seq in GV, PA, SCNT, and PEF samples. Chromosomes and IGV windows were randomly selected. B. Number of accessible regions detected in four groups. C. Coverage ratio of peaks in the genome detected by MACS2. 

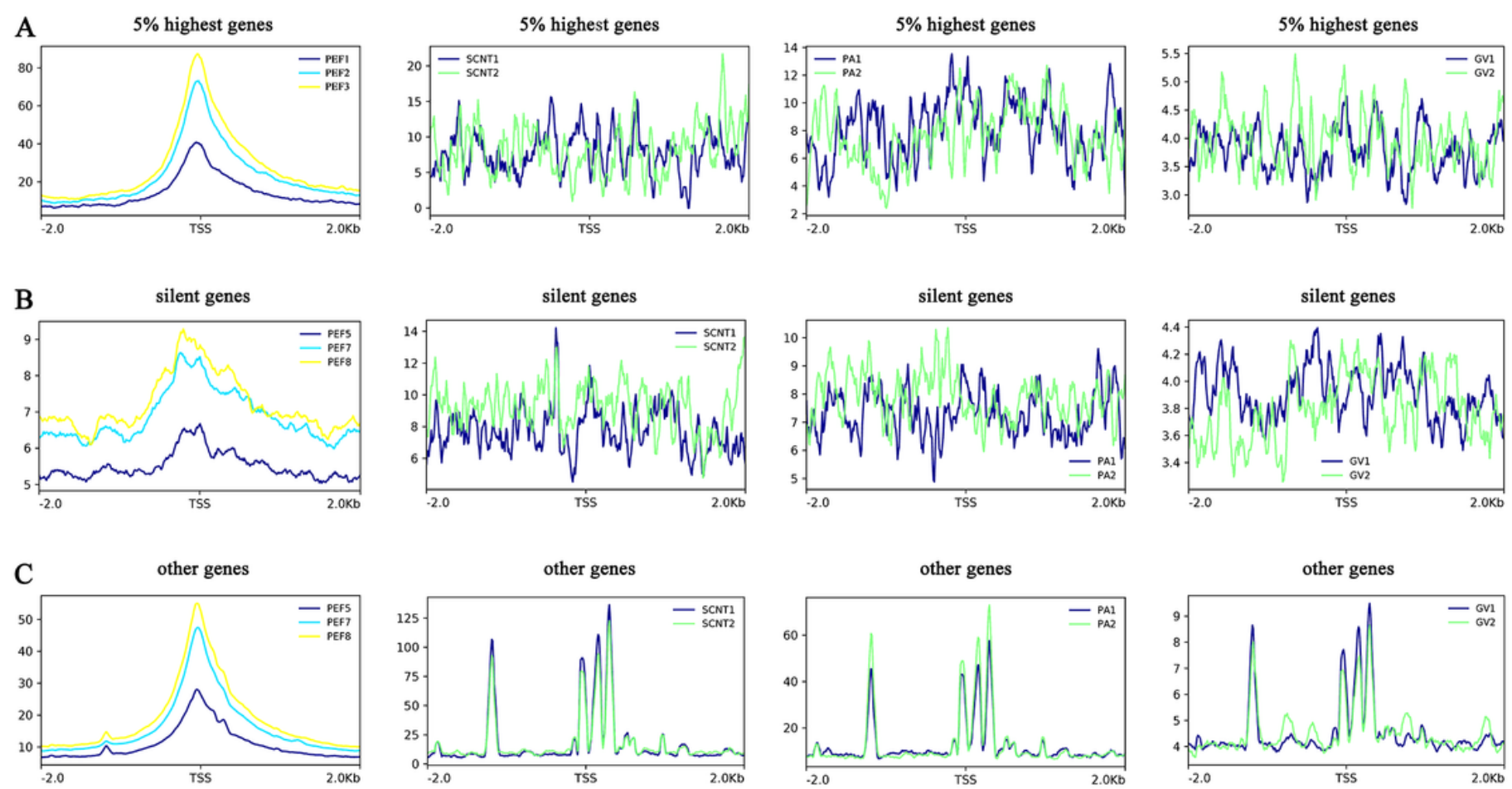

\section{Figure 3}

Dynamic changes in chromatin accessibility around TSSs during embryo reprogramming. Genes are classified into the top 5\% of genes expressed in PEF (A), silent genes (B), and other types of genes (C).
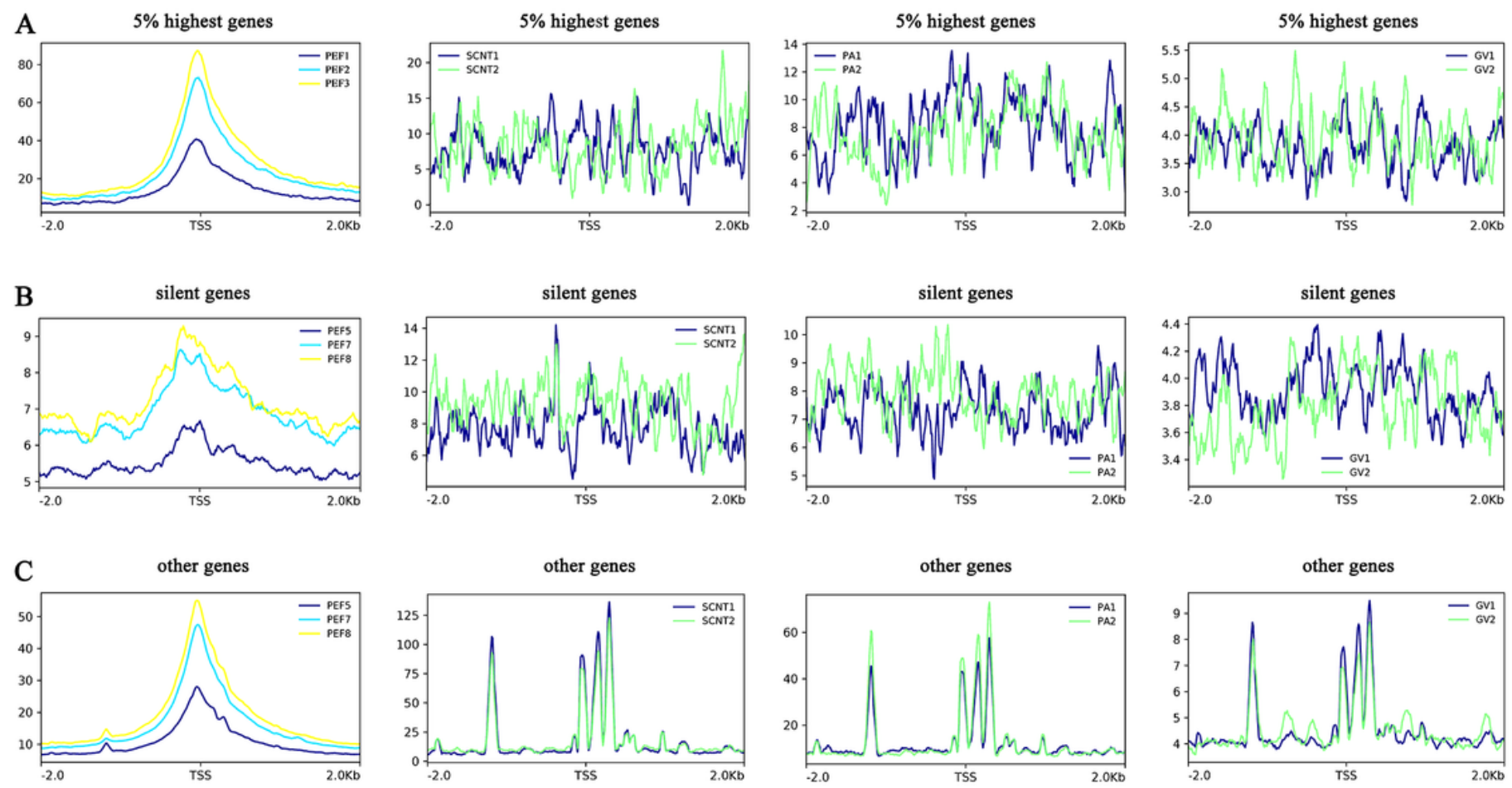

Figure 3 
Dynamic changes in chromatin accessibility around TSSs during embryo reprogramming. Genes are classified into the top $5 \%$ of genes expressed in PEF (A), silent genes (B), and other types of genes (C).
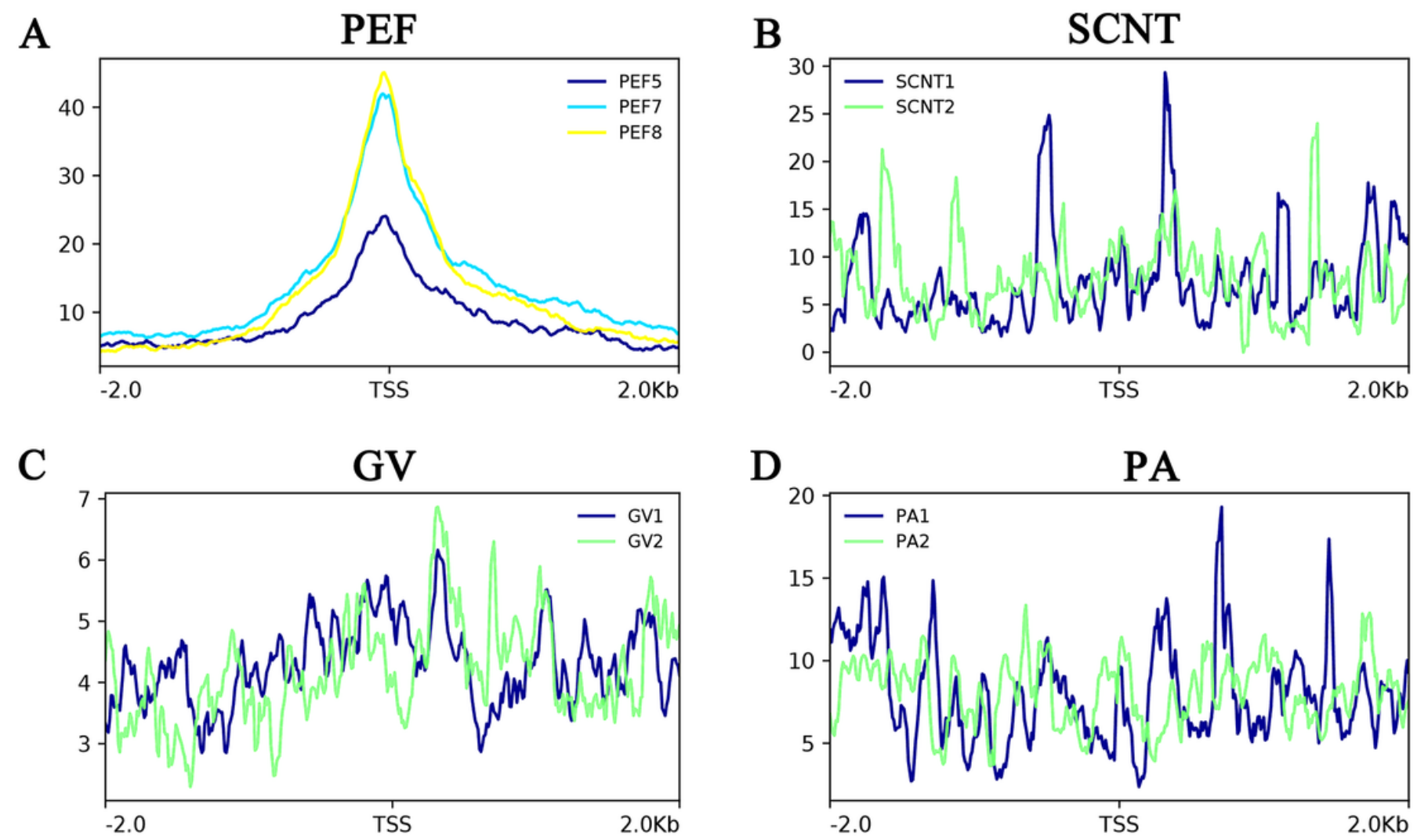

\section{Figure 4}

Dynamic changes in $\mathrm{X}$ chromatin accessibility during embryo reprogramming. The enrichment in ATACseq signals around the TSSs of X chromatin in PEF (A), SCNT (B), GV (C), and PA (D) are shown. 
A

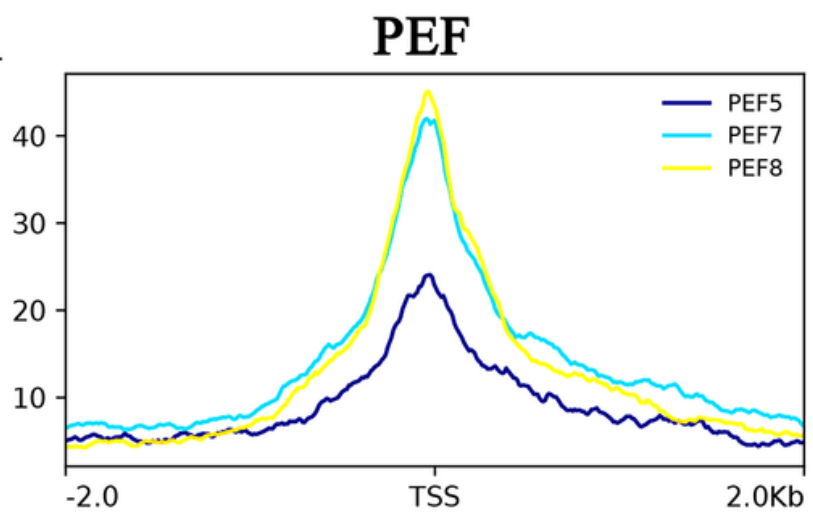

C

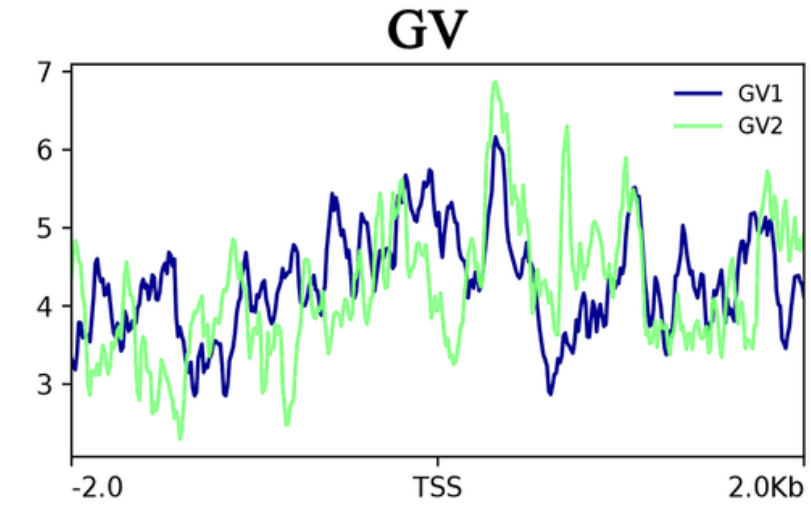

B

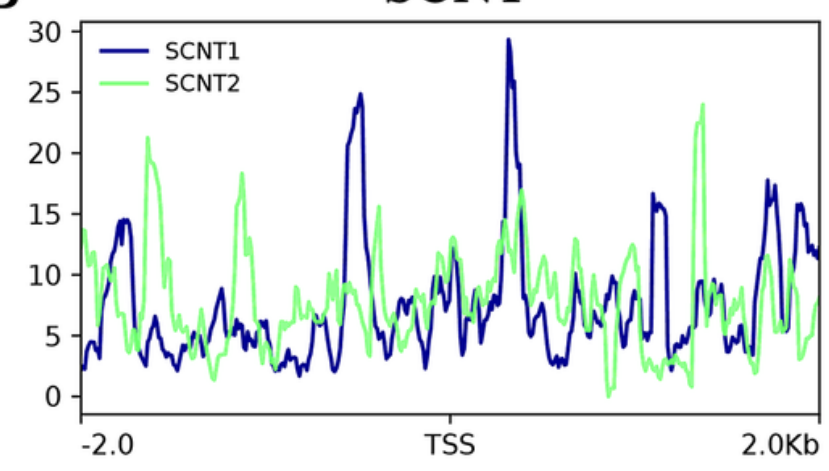

D

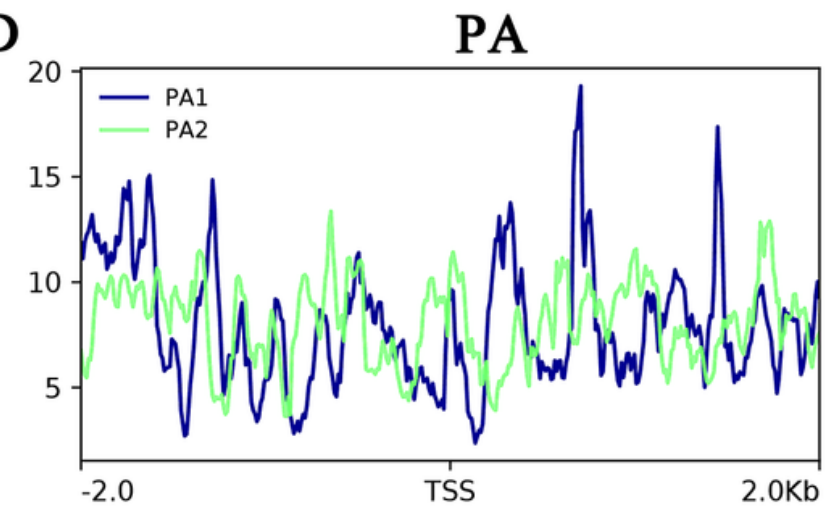

Figure 4

Dynamic changes in $\mathrm{X}$ chromatin accessibility during embryo reprogramming. The enrichment in ATACseq signals around the TSSs of $X$ chromatin in PEF (A), SCNT (B), GV (C), and PA (D) are shown. 
A

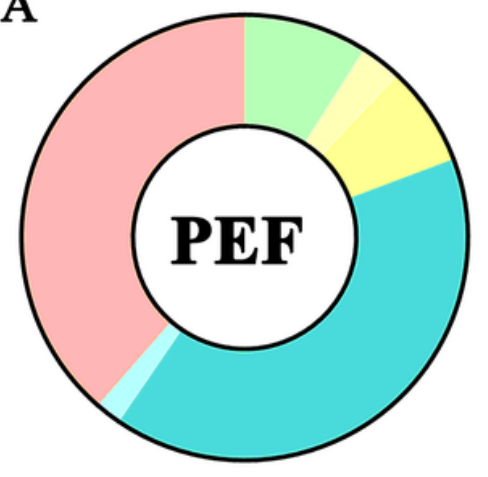

Promoter $8.99 \%$

5' UTR 3.05\%

Exon $7.22 \%$

Intron $40.27 \%$

3 'UTR 1.9\%

Intergenic $38.57 \%$
B

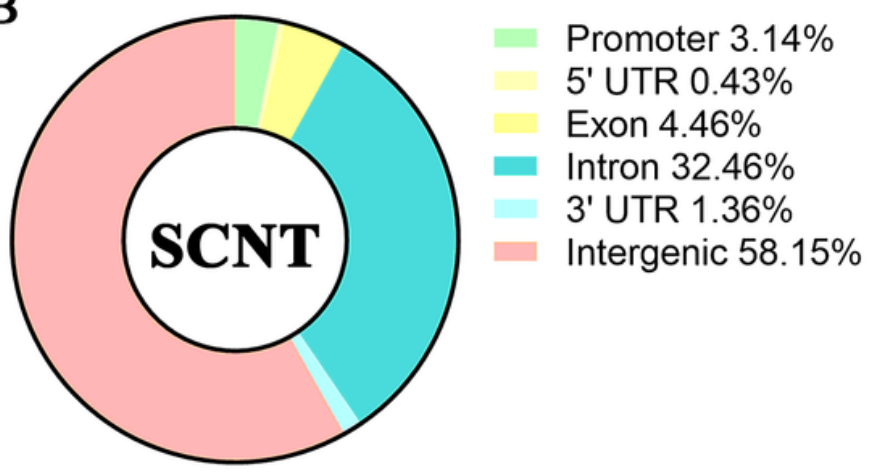

D

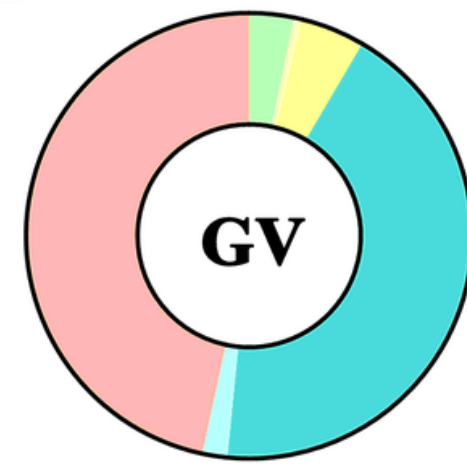

Promoter 3.24\% 5' UTR 0.59\% Exon $4.63 \%$ Intron $43.12 \%$ 3' UTR 1.79\% Intergenic $46.63 \%$

Figure 5

Distribution of chromatin accessibility sites in gene functional areas. The genome-wide functional regions were divided into the promoter, 5' UTR, exon, intron, 3' UTR, and intergenic regions for SCNT (A), PA (B), PEF (C), and GV (D). 
A

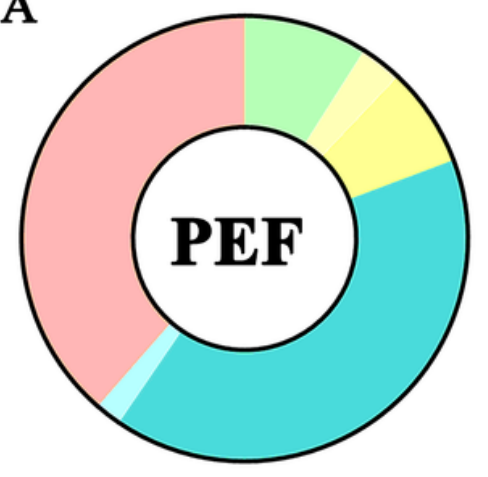

Promoter 8.99\%

5 ' UTR 3.05\%

Exon $7.22 \%$

Intron $40.27 \%$

3 'UTR 1.9\%

Intergenic $38.57 \%$

C

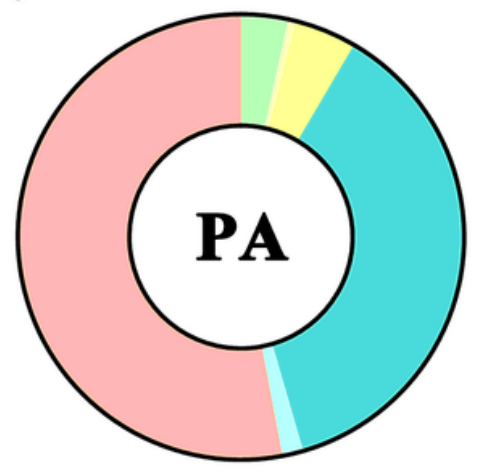

B

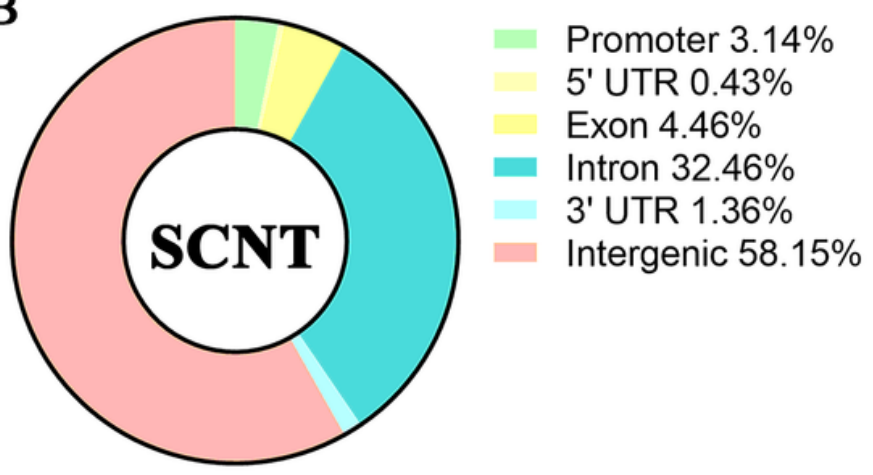

D

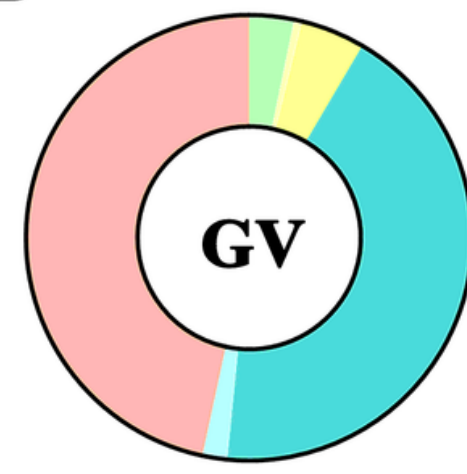

Promoter 3.24\% 5' UTR 0.59\% Exon $4.63 \%$ Intron $43.12 \%$ 3' UTR $1.79 \%$ Intergenic $46.63 \%$

Figure 5

Distribution of chromatin accessibility sites in gene functional areas. The genome-wide functional regions were divided into the promoter, 5' UTR, exon, intron, 3' UTR, and intergenic regions for SCNT (A), PA (B), PEF (C), and GV (D). 


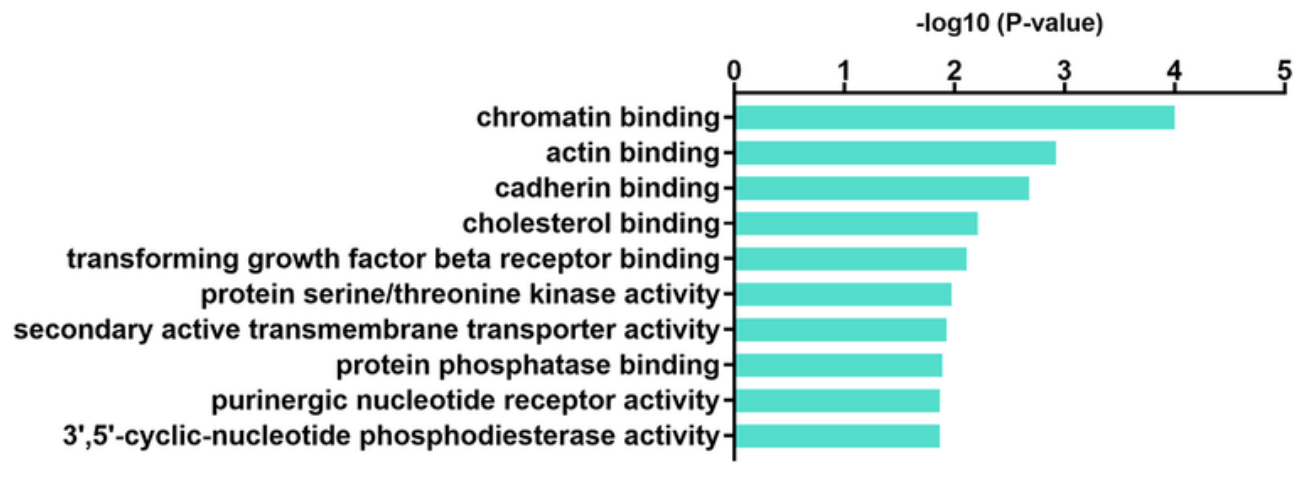

B

PA GO-Analysis-MF

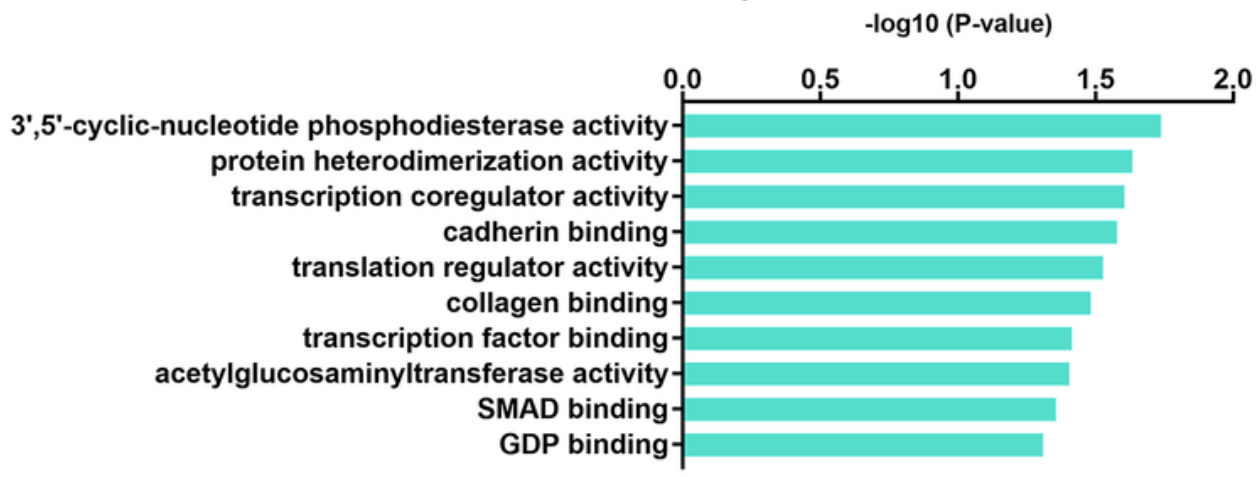

C

SCNT GO-Analysis-MF

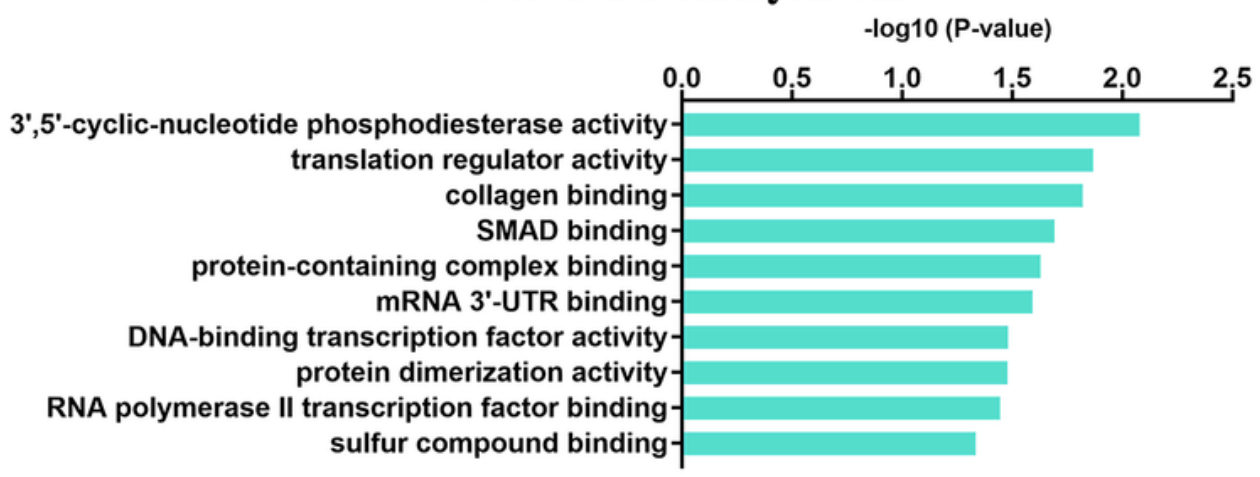

\section{Figure 6}

Molecular function of the GO analysis in GV oocytes, PA embryos, and SCNT embryos. Values on the xaxis correspond to the $-\log 10$ (P-value) for various $\mathrm{GO}$ terms; the top $10 \mathrm{GO}$ terms are shown. $\mathrm{GO}$ terms in peak-related genes were defined as significantly enriched if corrected p-values were $<0.05$. 


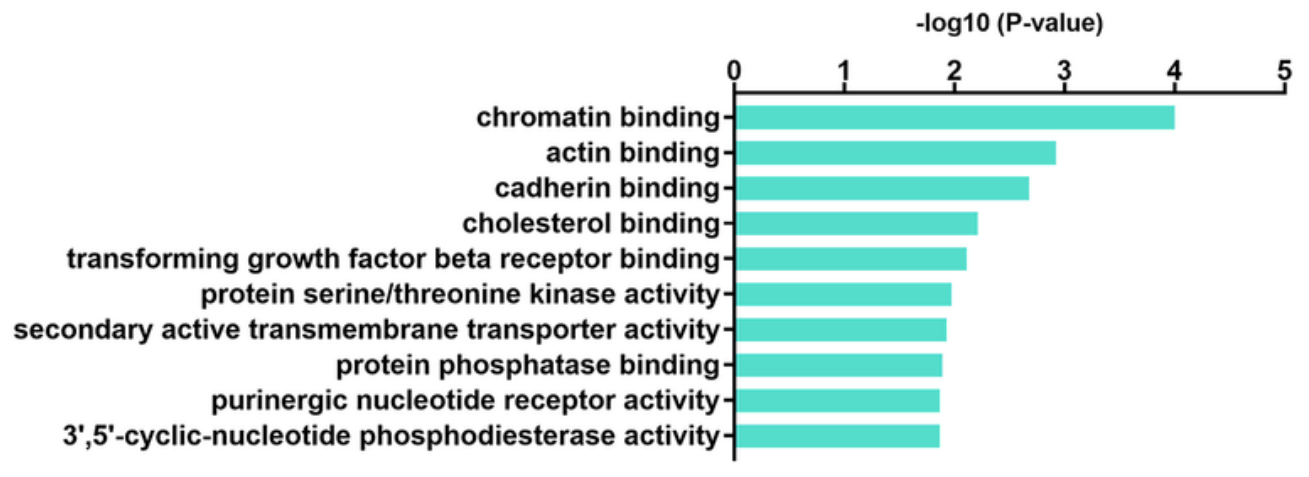

B

PA GO-Analysis-MF

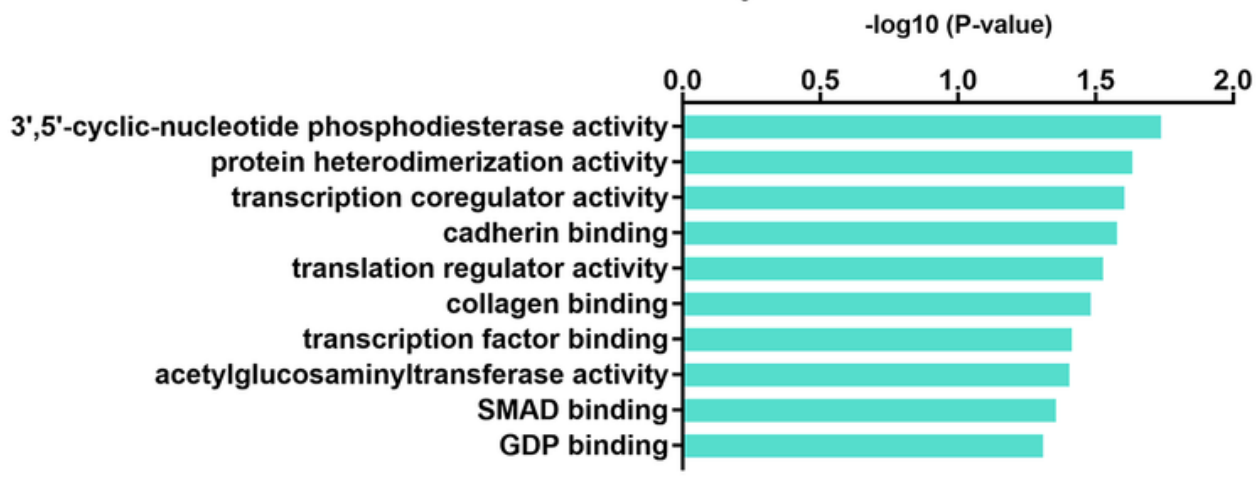

C

SCNT GO-Analysis-MF

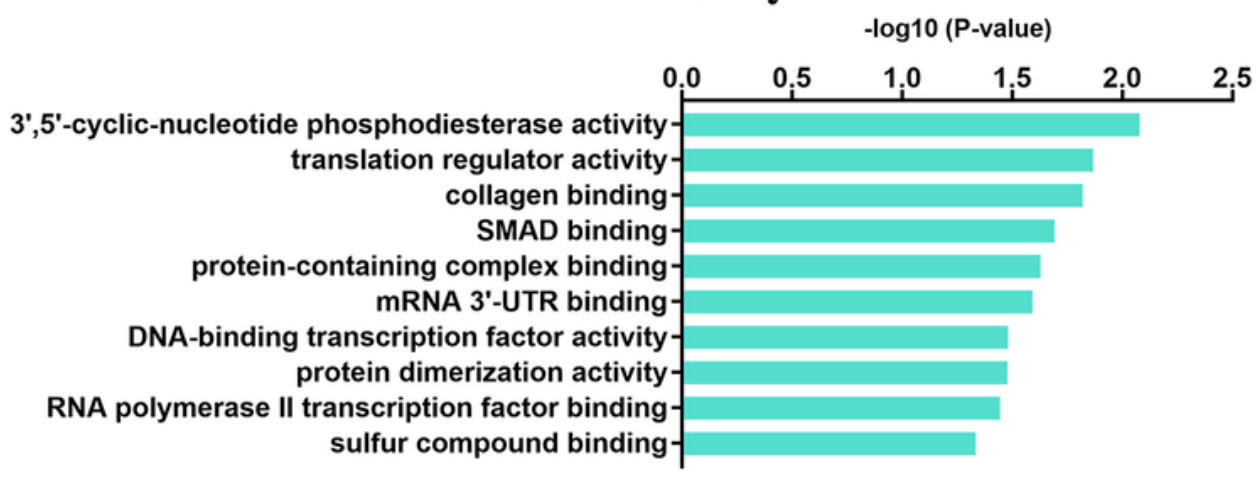

\section{Figure 6}

Molecular function of the GO analysis in GV oocytes, PA embryos, and SCNT embryos. Values on the xaxis correspond to the - $\log 10$ (P-value) for various $\mathrm{GO}$ terms; the top $10 \mathrm{GO}$ terms are shown. $\mathrm{GO}$ terms in peak-related genes were defined as significantly enriched if corrected $p$-values were $<0.05$. 


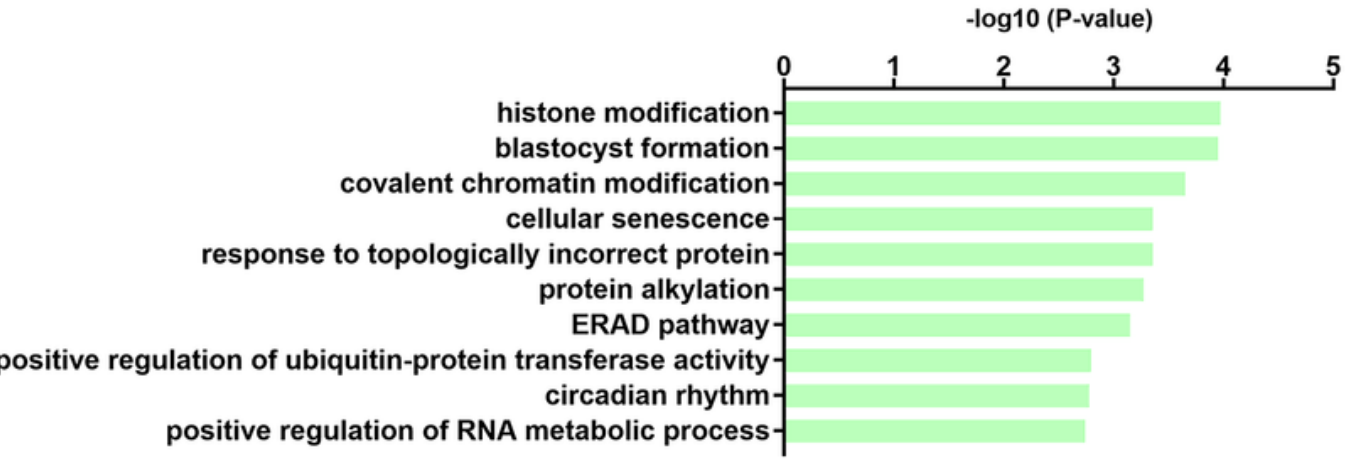

B

SCNT VS PEF GO-Analysis-CC

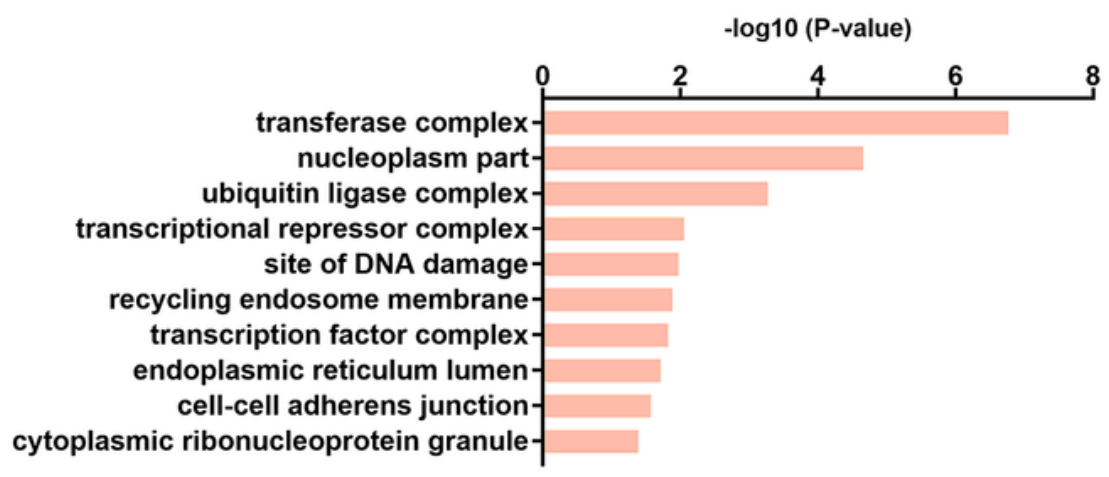

C SCNT VS PEF GO-Analysis-MF

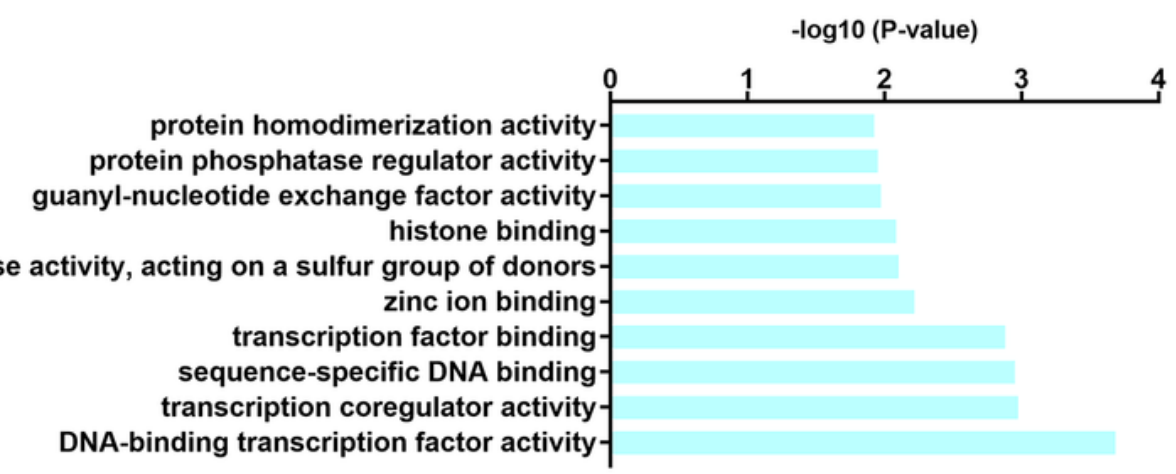

\section{Figure 7}

GO analysis of different peaks between SNCT and PEF. A. Biological process categories. B. Cellular component categories. C. Molecular function categories. Values on the $\mathrm{x}$-axis correspond to the $-\log 10$ (P-value) for various $\mathrm{GO}$ terms; the top $10 \mathrm{GO}$ terms are shown. $\mathrm{GO}$ terms in peak-related gens were defined as significantly enriched if corrected $p$-values were $<0.05$. 


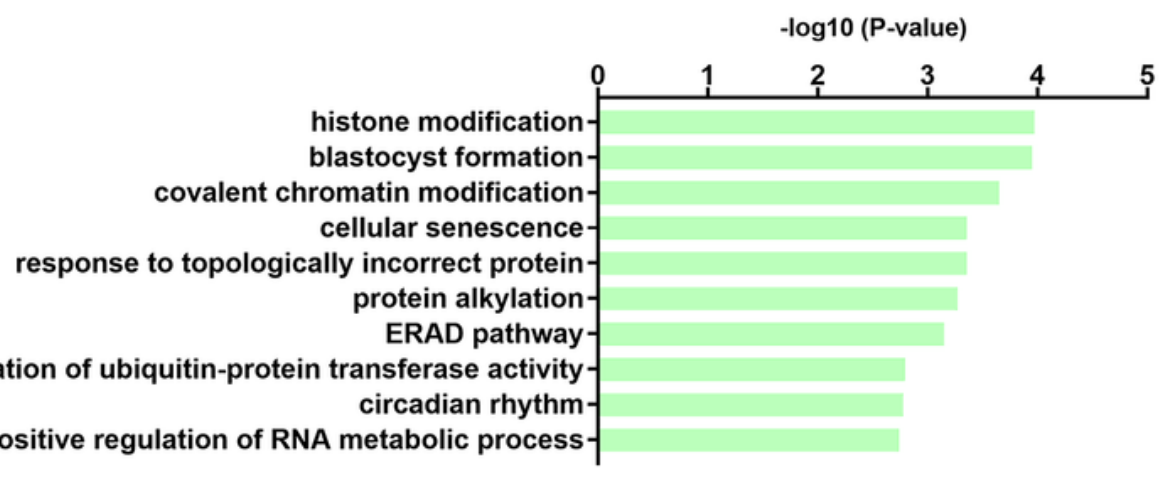

B

SCNT VS PEF GO-Analysis-CC

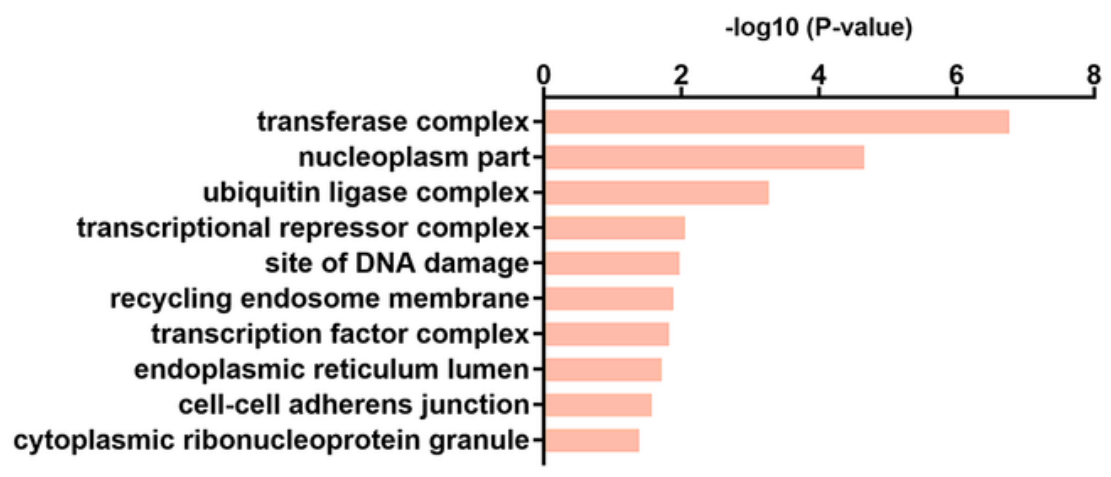

C

SCNT VS PEF GO-Analysis-MF

protein homodimerization activity-
protein phosphatase regulator activity-
guanyl-nucleotide exchange factor activity-
histone binding-
zinc ion binding-
transcription factor binding-
sequence-specific DNA binding-
transcription coregulator activity- .

\section{Figure 7}

GO analysis of different peaks between SNCT and PEF. A. Biological process categories. B. Cellular component categories. C. Molecular function categories. Values on the $\mathrm{x}$-axis correspond to the $-\log 10$ (P-value) for various $\mathrm{GO}$ terms; the top $10 \mathrm{GO}$ terms are shown. $\mathrm{GO}$ terms in peak-related gens were defined as significantly enriched if corrected p-values were $<0.05$. 